\title{
IOT DEVICES VS. DRONES FOR DATA Collection in Agriculture
}

\author{
Petkovics, I.; Petkovic, D. \& Petkovics, A.
}

Abstract: Modern agriculture requires constant plant, animal and machine monitoring so as to ensure optimal functioning. In the current age of agricultural robotization it is necessary to arrange for a suitable automated data collection system, primarily for plant and animal status which can provide the required data within a short time period. There are generally two alternatives for this purpose: a network of IoT (Internet of Things) devices, and drones (predominantly for plants) with different, mainly audiovisual, sensors that collect data when making a flight over the crops or animal habitat. Both alternatives meet the farmers' demand for increased success, while they also ensure decrease of costs. This chapter describes the characteristics of these two alternatives for data collection, highlighting the advantages and disadvantages of both solutions seen in their implementation.

Key words: IoT devices, drones, data collection, agriculture.
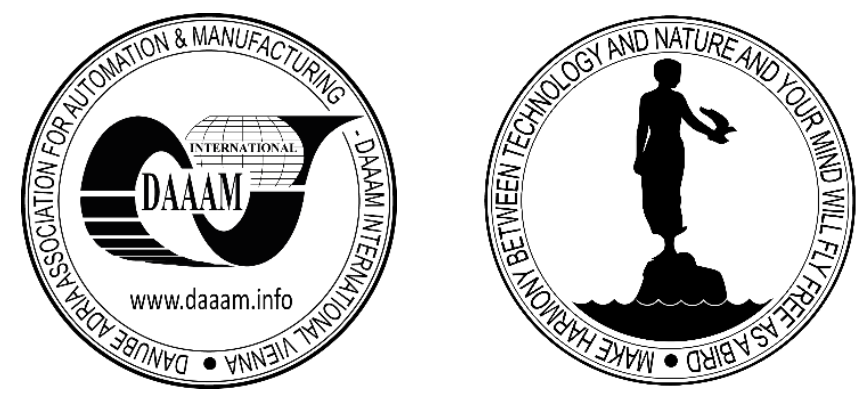

Authors' data: Univ.Assist.Prof. Dipl.-Ing. Petkovic, I[mre]*; Univ.Prof. Dipl.-Econ. Petkovic, Dj[erdji]*, Univ.Assist. PhD-stud. Dipl.-Ing. Petkovics, A[rmin $]^{* *} ; *$ University of Novi Sad, Faculty of Economics Subotica, Segedinski put 9-11, 24000, Subotica, Serbia, ** Budapest University of Technology and Economics, Muegyetem rkp.3, 1111, Budapest, Hungary, peti@ef.uns.ac.rs, pegy@ef.uns.ac.rs, petkovics@hit.bme.hu

This Publication has to be referred as: Petkovics, I[mre]; Petkovic, D[jerdji] \& Petkovics, A[rmin] (2017). IoT Devices vs. Drones for Data Collection in Agriculture, Chapter 06 in DAAAM International Scientific Book 2017, pp.063-080, B. Katalinic (Ed.), Published by DAAAM International, ISBN 978-3-902734-12-9, ISSN 17269687, Vienna, Austria

DOI: 10.2507/daaam.scibook.2017.06 
Petkovics, I.; Petkovic, D. \& Petkovics, A.: IoT Devices vs. Drones for Data Collec...

\section{Introduction}

The predicted percentage of agricultural product growth is a stunning $69 \%$ from the year 2010 to 2050 . This huge increase is to be generated primarily by the expected number of 9 billion inhabitants (Pardey et al., 2014). In order to meet these huge agrarian needs, the latest technologies are implemented, which are referred to by the terms 'AgriTech' or 'Precision Agriculture'. These terms indicate novel methods of running farms, based on monitoring, measuring, reacting to internal (including diseases, lack of nutrients and water) and/or external parameters (droughts, insects, rodents, wild animals) related to crops, as well as problems detected in game or livestock breeding. The main goal of this approach is to use the limited resources of the farms utmost efficiently so as to maximize the yield. The basic method in plant production is the minimization of plant change, i.e. maintaining crop health (Nixon, 2016).

Wildlife management and livestock breeding have the same afore-mentioned goals, only the techniques and technologies are specific given the non-stationary lifestyle. The implementation of precision agriculture involves a considerable amount of data and data processing. There are three groups:

1) Various types of recordings (visible and/or multispectral images) with geographic coordinates (geo-tagged images) on game and livestock, as well as crops during the vegetation periods, i.e. if necessary (this task is increasingly taken over by drones),

2) Equipment data: current data from the devices equipped with sensors (seeders, tractors, spreaders, combines, etc.) and

3) Management data: crop yield and other data provided by farm operators (Nixon, 2016).

There are two agricultural monitoring alternatives:

1) Network of intelligent sensors, i.e. IoT devices, in fact, IoT ecosystems (interconnected and connected to the Internet) and

2) Unmanned Aerial Vehicles (UAVs or drones) which, by recording and prompt or delayed analysis of the records, can provide similar or identical information as the IoT devices.

The implementation of an IoT ecosystem in Precision Agriculture is a state-ofthe-art solution based on communication, computer (Internet, cloud computing, big data and BI-Business intelligence) and sensor technologies (intelligent sensors and actuators). IoT is still in the phase of intensive development, though there are numerous examples of implementation in terms of specific solutions in agriculture. Based on the reports on realized projects in various fields of industry and society (Libelium, 2017b), it can be concluded that the use of intelligent sensor networks ensures immeasurable advantages by decreasing the costs of monitoring of game and livestock, as well as the quick discovery and prediction of diseases in the plant vegetation period. It must be conceded that, while the investment costs are also high, the savings are considerable (from 10 to 30 percent). 
The first drones were applied for military purposes long before the appearance of IoT devices. Drones as military weapons were robust, fast, durable, using a classic, or jet engine. Soon, however, the first models of a completely different construction made their appearance: fragile, light-weight, relatively slow, battery or electric enginepowered. These novel drones were primarily intended for the entertainment market, toy devices used for hobby: in photography and filming the environment from a new perspective, namely from high up. These flying vehicles evolved into useful appliances, making tasks easier to perform, faster and more precise, used in various areas of industry, especially in agriculture. Drones (also referred to as UAVs Unmanned Aerial Vehicles) have thus become a competition for IoT ecosystems mainly due to their low-level investment and flexibility when applied in practice. The widespread application of drones in agriculture, though, is increasingly difficult on account of legal regulations for their use. Even in states with the most developed culture and technology in the field of drone production, laws regulating the use of drones are currently being prepared.

This chapter first gives a description of the characteristics of the above-mentioned two alternatives for data collection and processing in agriculture, then continuous with outlining the differences, advantages and disadvantages of each alternative and presents guidelines for determining the right option for specific applications. This would offer assistance for choosing the optimal system for data collection and processing in agricultural companies/farms, in given situations and for specific tasks. There are certain instances when it cannot be readily and definitively decided which of the two described solutions should be chosen. The underlying reasons may be financial, professional-technical, geographic in nature; the implemented solutions must adhere to regulations, agreements and legal acts at the location of their implementation. For such occasions it is worth conducting a thorough search for different possibilities of data collection and processing on the agricultural market. These options will be outlined in the following section.

\section{Internet of Things - IoT}

The origin of this new concept 'IoT' was formulated as "computers everywhere" by professor Ken Sakamura (University of Tokyo) in 1984, also as "ubiquitous computing" by Mark Weiser (Xerox PARC) in 1988. The term 'Internet of Things' was coined by Kevin Ashton (Procter \& Gamble) in 1998 and developed by the AutoID Center of MIT from 2003. Ashton later described the IoT as "a standardized way for computers to understand the real world." (Zohu, 2012).

The instigation of developing IoT in Europe was a communication of the European Commission under the title "Internet of Things: An Action Plan for Europe" which was adopted on June 8, 2009 with a conclusion that stated in Point 4.1: "IoT is not yet a tangible reality, but rather a prospective vision of a number of technologies that, combined together could drastically modify the way our societies function in the next 5 to 15 years." and further in Point 3.11: "The Committee backs the Commission's plan for a proactive approach to ensuring that Europe plays leading role in shaping IoT so that the Internet of Things becomes an Internet of Things for People." (***, 2009). 
The IoT refers to fixed or mobile intelligent devices which can communicate and exchange data automatically via the Internet without human intervention. It is a concept through which the devices in one's environment will receive their addresses on the Internet and, using sensors and actuators, will become intelligent. That will enable every person to control the technology already in use in a novel way. (Petkovics \& Petkovics, 2014).

In order to achieve that, the IoT must have the so-called '5As' and '3Is' characteristics (Zohu, 2012). These two abbreviations refer to the '5As': anything, anywhere, anytime, anyway, anyhow, and the '3Is': instrumented, interconnected, and intelligent.

It must be highlighted that the IoT involves four well-known and independent fields of technologies, although this is rarely mentioned when referring to the IoT. These technologies are M2M (Machine to Machine), RFID (Radio-Frequency Identification), WSN (Wireless Sensor Networks) and SCADA (Supervisory Control and Data Acquisition). Usually the IoT is taken to cover WSN (which is referred to in the section title), but the more accurate description of the entire technology must not be so lenient and superficial. M2M uses devices connected in a network so as to monitor and record events of errors on these devices. RFID mainly uses radio signals for identification and object monitoring (for a certain central system) to which RFIDtags are attached. WSN represents the network of intelligent sensors (and sometimes, actuators, too) which are distributed in space so as to collect various data (and perform different actions by the actuator). SCADA refers to the autonomous monitoring system and control of complex systems (facility, factory) on the basis of wired short-range networks. Thus, the previously described technologies partly overlap (see Fig. 1. (Zohu, 2012)).

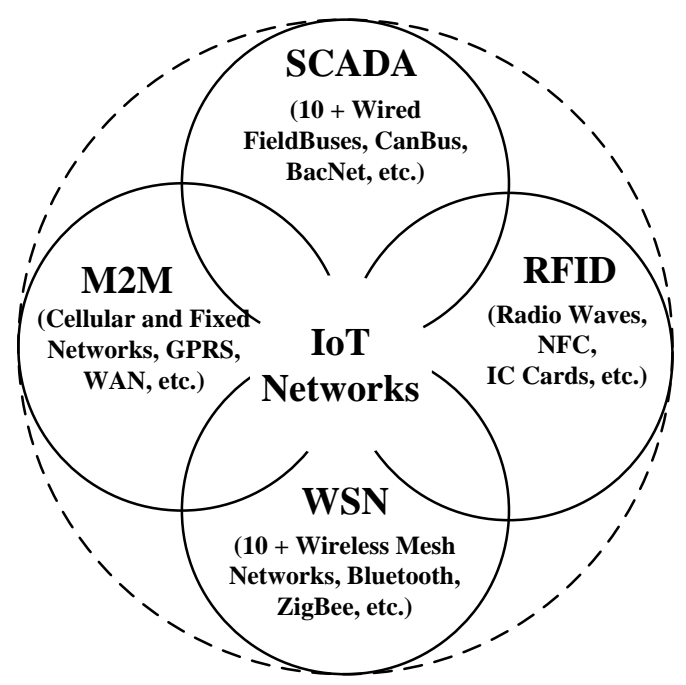

Fig. 1. Four technologies of the IoT system

The IoT modifies the functioning and use of the web. The reference Web 1.0 denotes one-way communication, i.e. transmitting certain content to the users so as to make it 'readable' via the browser. Web 2.0 ensured the upgrade of Web 1.0 to twoway communication with the help of new applications (blogs, social network services, 
video-sharing). The content generators for Web 1.0 and Web 2.0 were people, humans, whereas the appearance of the IoT introduced a new data generator: the machines. This qualitative change in the role of data generator, and especially the quantitative change in the amount of data, offers the basis for formulating the new model of using the World Wide Web with the reference Web 3.0.

Following the description of the IoT technology (without the formal, strict approach) in which, if no more, but at least the basic pillars (technologies) are complex 'structures' (of the IoT), then the strict definition of the Internet of Things can be attempted. Based on the short description given above, it can be stated that the IoT infrastructure covers a wide spectrum of technical, technological, network and infocommunication (ICT - Information and Communications Technologies) elements that must work in synch in order to ensure the basic services, thus it is virtually impossible to expect a single definition to be acceptable for all parties, researchers, experts, users, and contractors, as well. There are a number of definitions, statements, declarations and descriptions aiming to sum up the essence of IoT. It is therefore understandable that the definition of IoT can be neither short, nor simple. Perhaps the best definition among all of them is this longish formulation: "The Internet of Things is a plethora of technologies and their applications that provide means to access and control all kinds of ubiquitous and uniquely identifiable devices, facilities, and assets. These include equipment that has inherent intelligence, such as transducers, sensors, actuators, motes, mobile devices, industrial controllers, HVAC (heating, ventilation, and airconditioning) controllers, home gadgets, surveillance cameras, and others, as well as externally enabled things or objects, such as all kinds of assets tagged with RFID, humans, animals, or vehicles that carry smart gadgets, and so forth. Communications are via all sorts of long- and short-range wired or wireless devices in different kinds of networking environments such as Intranet, extranet, and Internet that are supported by technologies such as cloud computing, SaaS, and SOA and have adequate privacy and security measures, based on regulated data formats and transmission standards. The immediate goal is to achieve pervasive M2M connectivity and grand integration and to provide secure, fast (real time), and personalized functionalities and services such as (remote) monitoring, sensing, tracking, locating, alerting, scheduling, controlling, protecting, logging, auditing, planning, maintenance, upgrading, data mining, trending, reporting, decision support, dashboard, back office applications, and others. The ultimate goal is to build a universally connected world that is highly productive, energy efficient, secure, and environment friendly." (Zohu, 2012).

The motivation for introducing the IoT can be classified from the aspect of entity, i.e. the medium which implements the given solution (Petkovics et al., 2013):

\section{Organization}

1.1. collection and storing data monitored in real time using an IoT device,

1.2. efficient resource management of the interconnected networks (using the provided sensors),

1.3. processing and analysis of data from IoTs stored in databases or data warehouses at the organization computational center, the cloud, the local server (gateway, to be elaborated on in the section below) or the node itself, to which the sensors are attached (further explanation to be given below). 
1.4. current data and its real-time processing and analysis in terms of indispensable reports represent the basis for making good business decisions, and solid business decisions ensure the functional efficiency which contributes to the maintainable development of the organization,

1.5. realizing the monitoring of device operation, monitoring of plant development and status, as well as animal movement relevant to production and functioning of the organization ensure the decrease of business costs,

1.6. faster development of products and services, because of the interconnected devices and their communication with each other, etc.

2. Human beings

2.1. monitoring the organisms' vital functions,

2.2. monitoring the parameters of the patients' diseases remotely with the option of taking the required actions if necessary,

2.3. tracking the availability of vehicles in urban transport and carpooling (Grgurevic et al., 2015) in real time,

2.4. decrease and control of heat and electrical energy consumption,

2.5. surveillance and security of private property, etc.

3. Government and society

3.1. realization of the smart city (monitoring and control by distribution of energies, water, information channels, emergency services, etc.)

3.2. realization of self-guided cars and their integration into the smart city,

3.3. monitoring and security of social property, etc.

\subsection{Architecture of the IoT system}

Based on the outlined characteristics and definitions of the IoT, the following three layers of architecture of the IoT system can be determined (Davies, 2015):

1. Device layer with integrated sensors,

2. IoT device network layer - control and data collection (and temporary storing) provided by the device sensors - Gateway layer, and

3. Platform layer which performs permanent data storage, as well as processing.

The devices with integrated sensors may contain certain or even all elements of microcomputer configuration: processor, memory, external memory, input-output devices, operating system and applications. These are often referred to by the term 'node'. The devices/nodes are connected in a local network (via cables - Ethernet, HomePlug, HomePNA, HomeGrid and LonWorks networks or wireless lines - WiFi, Bluetooth, Bluetooth Low Energy, RFID, NFC, Xbee, Zigbee, Z-Wave, Meter-Bus, Wireless M-Bus) in which the gateway takes the role of the local server. For networks that cover a wider geographical area, mainly the already existing mobile networks (GSM, 3G, LTE, WiMAX, GPRS, SIGFOX, NeulNET), as well as satellite connections are used. For certain implementation, synchronous communication of the IoT devices with the gateway is not required, thus in order to save energy, devices are implemented for the realization of asynchronous communication on the principle of scanning local IoT nodes from the local server (gateway) side.

It will soon be proven that a great amount of data collected by the IoT devices is relevant and necessary, while numerous people and organizations are also ready to pay 
for this data. Moreover, it is not the data itself that is the most important, but the information that can be extracted after processing. This is the philosophy of cloud computing (CC). The business (service) model of the IoT system could be constructed on the principle of the accepted service model for CC. It is well-known that $\mathrm{CC}$ has three basic services: (1) Infrastructure as a Service (IaaS), (2) Platform as a Service (PaaS) and (3) Software as a Service (SaaS). In recent years, a new service related to the IoT systems has been mentioned increasingly often, this is Sensing as a Service $\left(\mathrm{S}^{2} \mathrm{aaS}\right) . \mathrm{S}^{2} \mathrm{aaS}$ offers data collection by IoT sensors of general interest that are valuable for many people and organizations. However, despite the visible similarities between the $\mathrm{CC}$ and $\mathrm{S}^{2} \mathrm{aaS}$, the supporters of this new $\mathrm{S}^{2} \mathrm{aaS}$ tend to disregard the architecture of the IoT system (Perera, 2017).

The IoT system realized for a specific purchaser (organization) does, in fact, have the above-described architecture (Davies, 2015), but $\mathrm{S}^{2} \mathrm{aaS}$ is intended for a larger number of users, thus it must or should have an architecture that ensures the IoT services on three layers (as CC): (1) raw IoT data located in the cloud (corresponding to IaaS), (2) raw IoT data in the cloud with development environments/ program languages and/or prepared software for processing/analysis (similar to PaaS), and (3) IoT data processed/analyzed in several ways (using the most often required algorithms/processing by the user), with the results of processing the IoT data located in the cloud (just like SaaS). The users could choose for themselves the processed IoT data prepared by the provider based on their specific requirements.

The architecture of the IoT system means that if it is offered as a service ( $\left.\mathrm{S}^{2} \mathrm{aaS}\right)$, it ought to/should have the following architecture (layers):

1. Device/node layer with integrated sensors,

2. Gateway layer - controlling the network of IoT devices and collecting (as well as temporarily storing) the data,

3. Stored raw data layer in the cloud (IaaS cc service),

4. Stored raw data layer in the cloud with software for development and/or processing (PaaS CC service), and

5. Processed IoT data layer (SaaS CC service).

\subsection{Types of data sources of IoT systems}

The sources of data of the IoT systems are, naturally, the sensors. The number of sensors in the nodes of the IoT systems may vary. The intelligence of the nodes is the function of their inherent ICT resources. The sensors in the nodes can be classified into three groups (Linthicum, 2017):

1. Asynchronous sensors,

2. Synchronous sensors, and

3. Intelligent sensors.

All types of sensors require a different infrastructure of the environment and different managing/handling/control.

Asynchronous sensors are not able, as their name suggests, to initiate communication. These sensors must first be activated, and then they can be requested to transmit the collected data. For the collection and processing of IoT data, the IoT 
application itself must perform these two actions so as to obtain the required data. These are low power sensors placed at remote locations.

The synchronous sensors are permanently active and they realize one-way, synchronous communication: they continuously generate and send data to the IoT application. It must absorb the data stream, separate the data and perform their basic function (data storing and/or processing). It is vital that a suitable communication channel is provided for data security during transmission that has adequate transmission band width/speed.

Intelligent sensors/devices are such active sensors/devices that perform bidirectional synchronous communication with a wide range of possibilities. These options that, in fact, make these sensors/devices intelligent (or dynamic, according to (Linthicum, 2017) include the following: modification of the generated data (this characteristic naturally refers to those devices with multiple sensors - it is unrealistic today to expect a temperature sensor to measure atmospheric pressure, as well, on demand), modification of the measured data format, modification of the communication channel frequency, modification of the security coding for data transmission (e.g. to blockchain /Bitcoin (Noyen et al., 2014)), performing automatic updates of the software, etc. These sensors/devices are most complex in nature and are thus preferred in future IoT applications.

\subsection{IoT system hardware}

When presenting the necessary hardware for the IoT system, it is sufficient to consider only the first two layers of IoT architecture: device layer and gateway layer. In larger networks of devices/waspmotes, the classical routers can also be implemented in communication, which are located between the gateway and waspmote. The third layer (platform layer) and, if one considers the five-layer architecture for $\mathrm{S}^{2} \mathrm{aaS}$, then the other three layers, have the traditional role of data processing, which can be realized by the IoT platform in its own environment or on premise, or with the help of CC.

The device layer covers a growing number of sensors. One of the most famous manufacturers of IoT sensors, Libelium, has more than 120 types of sensors. These sensors are placed at the location of data collection in groups. The suppliers of IoT devices thus have readily prepared devices (Libelium calls these 'waspmotes') with a greater number and combination of sockets for the individual sensors of all possible fields of application. The devices with their attached sensors collect the data that was collected by the sensors and transmit it to whichever gateway it belongs to. Communication ranges from $100 \mathrm{~m}$ to even $100 \mathrm{~km}$, depending on the protocol, i.e. which network is used for communication. For greater distances, the cellular network must be used, though it is rarely implemented due to the high costs involved. Instead, currently they use the LPWAN network (Low-power wide-area network) increasingly often, featuring a range of up to $50 \mathrm{~km}$. Other characteristics of this network are also suitable for the IoT system: low energy consumption, long life, cheap radio module, etc. The sensors differ in their type and amount of data they collect, as well as in the data collection interval and charging.

The role and necessity of the gateway layer can be explained as follows (Konsek, 2015): the sensors usually have one or two integrated communication protocols. 
Sensors by different manufacturers use different pairs of protocols with a limited range of communication. The implemented protocols in most of the sensors do not have the ability to communicate with the larger networks (Internet, WAN). The importance of the gateway lies in its communication with the sensors (using their protocols), further, in the purification and unification of the data received from the sensors, in the aggregation of the data, implementation of security coding, preparation of communication packages of messages and transmission of these to the processing center. The gateway must decide if the data from the synchronous or intelligent sensors must be temporarily stored in the gateway device itself, and if so, then it must also time the transmission of this data. In certain time-critical IoT applications (for real-time operations), the data is not transmitted to the processing center, but processed in the gateways. In these cases, the gateways' processing power must be such that they can ensure prompt reaction, if necessary. Moreover, the gateway is also vital when tracking and controlling the operation of the sensors (nodes), as individual monitoring of the nodes is indispensable. The gateway devices are rarely equipped with their own integrated sensors, or if they are, these are generally temperature sensors, for tracking the working temperature in the gateway's housing, and/or a GPS device, for transmitting data to the processing center.

Installing and updating the software at the gateway is performed in these four ways (Konsek, 2015):

1. Factory bootstrap,

2. Server-initiated bootstrap,

3. Client-initiated bootstrap, and

4. Updates over-the-air

The first option does not offer the possibility of software update at the gateway (as is the case with the majority of gateways). The second and third options are performed 'within', where the IoT system is installed and operating, while the fourth option is used mostly only for security fixes. Libelium has lately been combining the third and fourth options of software update in their devices equipped with sensors waspmotes (Libelium, 2017a).

\subsection{Models of IoT data procession}

IoT data procession can be achieved by one of the four methods listed below:

1. On premise IoT platform

2. IoT platform in the cloud $(\mathrm{CC})$

3. Edge Computing (EC)

4. Fog Computing (FC)

The on premise IoT platform as a processing model is an old-time classic solution for data processing at the ICT resources found at the environment of the organization that intends to use the information and knowledge gained from IoT data processing. The IoT platform in the cloud is a realization of IoT data processing with all advantages, disadvantages and risks, characteristic of CC model (Petkovics \& Tumbas, 2014).

In real-time decision making situations, the delayed data processing by the sensor devices and/or the delayed obtaining of results is not acceptable. In such cases the IoT 
data must be processed on premise. This is the underlying notion of the processing method called EC. The EC concept, which is also referred to as Mesh Computing, places applications, data and processing at the logical extremes of a network rather than centralizing them. Placing data and data-intensive applications at the edge reduces the volume and distance that data must be moved (***,2016b). For conducting such data processing, the sensor devices (note, these are not regular waspmotes) must feature suitable ICT performances (processing power, memory, etc.) in order to achieve quick and efficient data processing of local IoT data. The reason for implementing EC can also be the protection of sensitive business data, whose security during transmission and in the cloud cannot be fully guaranteed. Another motivation for implementing EC is that a large amount of IoT data placed in the cloud can lead to the sudden rise in costs of renting the $\mathrm{CC}$ services due to the increase in memory space, as well as the integration of this data with the organization data.

FC is an architecture that uses a single or large number of collaborative end-user clients or near-user edge devices to carry out a substantial amount of storage (rather than stored primarily in cloud data centers), communication (rather than routed over the Internet backbone), and control, configuration, measurement and management (rather than controlled primarily by network gateways such as those in the LTE (telecommunication) core) (Perera et al., 2017). "Fog computing is a system-level horizontal architecture that distributes resources and services of computing, storage, control and networking anywhere along the continuum from Cloud to Things, thereby accelerating the velocity of decision making. Fog-centric architecture serves a specific subset of business problems that cannot be successfully implemented using only traditional cloud based architectures or solely intelligent edge devices." (***, 2016b). FC expands and complements the capabilities of the CC model, while it also decreased processing costs compared with data processing costs in the cloud. EC domain is a subset of FC domain, as seen in Fig. 2 (***, 2016c).

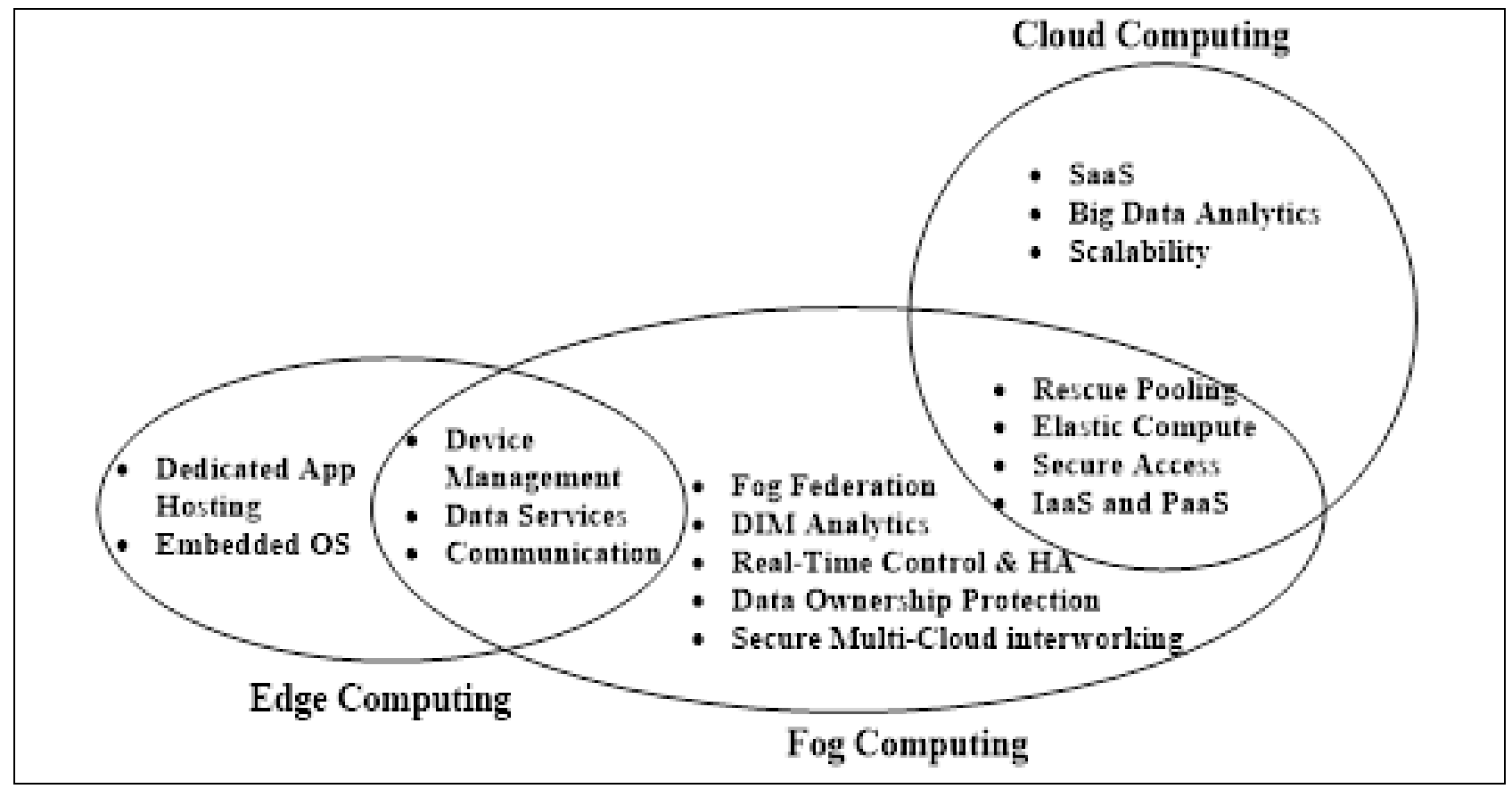

Fig. 2. Comparison of Cloud Computing, Fog Computing and Edge Computing 


\section{Drones}

The market of civilian drones is currently experiencing a rapid surge. Their implementation covers a wide range of tasks: monitoring power lines (electric power lines, oil pipelines, gas lines), monitoring plant development and health status (in forests, plantations, in agriculture, in greenhouses, etc.), monitoring of game and livestock movement, traffic monitoring and monitoring of private properties, tracking movement of individuals, observation of train track conditions, drawing precise maps, package delivery, human transport, providing Internet connection, etc. Considerable overlaps are perceived when comparing the application of drones and IoT devices.

The first drones were implemented before the appearance of IoT devices; they were used for military purposes. Those were primarily combat drones, robust and fast in nature, durable, engine or jet engine powered. The first civilian drones were completely dissimilar to the military drones precisely because of their different implementation: relatively slow, light-weight, fragile and mainly electric engine powered. There are constantly newer and newer types and models of unmanned aerial vehicles. In 2016, the number of companies dealing with drones was 711 in 49 different states $(54 \%$ of these are located in North America, while 30\% are found in Europe). These companies can be classified based on their products/services: "Platforms (29\%), Components and Systems (16\%), Services (20\%), Universities and Research Programs (10\%), Software (7\%), News/Media/Blogs (6\%), Coalition/Initiatives (5\%), Conferences and Events (3\%), Operator Marketplaces (2\%), Drone Insurances (2\%), User Groups/Networks (1\%)" (***, 2016d). The percentage of drones implemented in agriculture is relatively low. However, data analysis from the year 2015 highlighted that the value of businesses and labor in agriculture industry that may be replaced by drones/drone powered solutions amounts to 32.4 billion dollars (***,2016a). Thus a significant rise in drone application is expected by 2020 .

\subsection{Types UAVs}

The basic classification of unmanned aerial vehicles is based on the mode of flight realization:

1. drones - fixed-wing aerial vehicles,

2. copters/multi-rotor drones, and

3. hybrid drones.

Fixed wing drones are relatively weighty as they have heavy batteries, they have also greater load bearing capacity, therefore are capable of carrying a larger number of sensors, further, they achieve greater speeds and have longer flight time, if compared with copters. They are primarily suitable for farms with greater areas and long plots of land. The fixed wing drones have embedded software for flight planning and control based on GPS navigation, as well as Google maps. In comparison with copters, their prices are significantly higher. Moreover, these drones have great space requirements, a runway of $20 \times 50 \mathrm{~m}$ for landing, while some of the models even need a ramp for takeoff.

Copters, on the other hand, have slower flight speeds, their maximum flight time is approximately half than that of fixed wing drones. Their payload is also relatively 
small, sufficient for only few sensors, hence, on average, it takes copters twice as long to collect the same amount of data as the data collection by fixed wing drones. Copters do not require a takeoff ramp, they can float in one spot, unlike fixed wing drones. Copters are intended for smaller, more limited spaces and surfaces that are not easily accessible. They are also considerably cheaper than fixed wing drones (Petkovics et al., 2017).

Hybrid drones can switch their flight/operation mode: in one mode they are able to float, just like copters, while in the other mode, they fly in the same manner as fixed wing drones $(* * *, 2017 \mathrm{a})$.

\subsection{Architecture of IoT systems and their ICT elements in drones}

Certain experts believe that drones only cover mobile sensors or mobile gateways with sensors. According to them, drones are specific solutions of the device layer or gateway layer. This may be true to some degree, since the IoT system operating in the drones can also be three-layered or five-layered, as previously detailed in Section 2.1. Nonetheless, drones are distinctive vehicles as they must contain sensors used exclusively for navigation. The operations of these sensors, as well as the drone's navigation program are, in fact, necessary (useless from the point of view of data collection) general costs. The copters commonly include only devices with sensors, or possibly gateways, for the purpose of data transmission via the communication channel towards the processing center at the IoT platform. Due to their greater payload capabilities, the fixed wing aerial vehicles can be furnished with the complete ICT equipment for data processing from sensor devices, which means that the processing results are available immediately after the flight to authorized users. In the majority of cases for both drones with fixed wing and copters, the data from the sensors is stored on a local medium, while its processing is performed on the user's computer or in the cloud. The real-time data processing on the drone itself is required only for navigational purposes.

\subsection{Types of data sources, i.e. types of sensors used in drones}

Drones are exceptional devices also due to their specific types of data sources. Useful/user data collection occurs exclusively via synchronous sensors. This is explained by the fact that, when the drone is not airborne, there is no need to activate an asynchronous sensor. However, when it is in flight above the land for observation, the embedded sensors must operate continuously and the collected data must be stored until the end of the data acquisition.

Synchronous sensors in drones fall under one of these two groups: (1) flight and navigation sensors, and (2) camera sensors. The flight and navigation sensors are devices that generate their own signal for task execution, consisting of the signal source (light signal or signal in the invisible wave spectrum), receiver signal (the camera), and the controller on a board. The sensors-devices that only detect, or detect as well as analyze the signal, do not have the component of the signal source (generator).

The flight and navigation sensors are controlled by the drone status, flight parameters, the sensors also aid the navigation and monitor the immediate or farther environment of the drone in order to detect and avoid unexpected obstacles (crash 
avoidance) in the mapped flight trajectory. These sensors include the vision sensor, ultrasonic rangefinder, LIDAR sensor, gyroscope, accelerometer, 1D rangefinder, 2D rangefinder, odometer, IMU (Inertial Measurement Unit - with multi-axis magnetometers/compasses), tilt sensor (with gyroscopes and accelerometers), current sensor, magnetic sensor, engine intake flow sensor, GNSS (Global Navigation Satellite System - using the GPS, GLONASS, Galileo or BeiDou system), GPS (Global Positioning System), DGPS (Differential Global Positioning System - enhancement to GPS that provides improved location accuracy, from the 15-meter nominal GPS accuracy to about $10 \mathrm{~cm}$ in the case of the best implementations), ToF (Time-of-Flight) Camera Sensors, among others.

Cameras must have at least a 12 megapixel resolution with different frequency ranges of recording, as they present the basic useful load of drones used for monitoring in agriculture. By setting the suitable filter, one can use a common camera to take NIR (near-infrared) shots, although these shots may not be sufficiently high-quality, reliable or accurate in all moments of implementation (Delaporte, 2015). The recordings at three to five frequent ranges are quite satisfactory for use in agriculture, excellent options for the following tasks (cited verbatim from Corrigan, 2017):

- Identify pests, diseases and weeds. Optimize pesticide usage and crop sprays through early detection.

- Provide data on soil fertility and refine fertilization by detecting nutrient deficiencies. Help with land management and whether to take ground in or out of production or rotate crops etc.

- Count plants and determine population or spacing issues. Estimate crop yield.

- Measure irrigation. Control crop irrigation by identifying areas where water stress is suspected. Make land improvements such as install drainage systems and waterways based on multispectral data.

- View damage to crops from farm machinery and make necessary repairs or replace problematic machinery.

- Survey fencing and farm buildings.

- Monitor livestock.

The implementation of two-three cameras with adequate frequency ranges is rarely used in recent times. Instead, the trend is to use multispectral sensors with three to six sensor devices. These sensors are ultra-light weight (150 g to $200 \mathrm{~g}$ ) and superior to classic cameras for the same purpose, though it must be pointed out that the highend models are quite expensive (Nebiker et al., 2016). The multispectral sensors cover a frequency range that corresponds to visible light. There are, however, also cameras in the thermal infrared frequency range that can see hotspots and measure changes in land and plant temperature over time. These cameras can also detect objects that neither the human eye nor the multispectral sensors can: the presence of water due to its cooling effect, which can be helpful in spotting crop damage caused by drought and/or seasonal issues. With the help of these cameras that measure temperature differences it is possible to calculate CWSI (crop water stress index) in order to detect/predict water stress in plants (Nixon, 2017). 
Petkovics, I.; Petkovic, D. \& Petkovics, A.: IoT Devices vs. Drones for Data Collec...

\subsection{IoT data processing models}

Four IoT data processing models were described in Section 2.4., though in fact, in the process of drone data collection and processing, only two of these models are used: the on premise IoT platform and the IoT platform in the cloud. The IoT platform in the cloud is a processing model that is usually executed from the user computer. In rare situations the drone itself transmits the collected data from the sensors into the cloud. The users generally have some data processing software on their computer. That software is normally delivered along with the drone.

\section{Discussion}

Regarding the implementation of IoT devices and UAVs, it can be stated that they are both suitable solutions for data collection and processing in agriculture. Data processing is carried out by ICT resources that are closely connected with IoT devices and drones for data collection. The implementation of IoT systems has highly useful features, such as modularity (installation of a new IoT device into the network), robustness/fault tolerance (failure of one sensor does not impact the functioning of the entire network), flexibility (the sensor network does not have a fixed architecture), and low power consumption (of all the devices in the network architecture). The IoT devices are rather expensive. Fixed points for installation of IoT devices in the fields are determined by experts.

The drones are becoming increasingly more appreciated, which naturally leads to their wider use in agriculture. In order for them to work, it is not necessary to set up and install a large number of smart sensors scattered over large areas, monitor their operation, replace faulty IoT devices and obtain secure Internet connection for online transmission of the collected data. Drones, unlike IoT devices, are not fixed to a geographic location, the data collected by their sensors and cameras are suitable for different kinds of analysis (the recordings of the thermal sensors can be analyzed from the point of view of water presence on the area, surface distribution of soil temperature, plant temperature, and presence of animals on the given territory). Given the fact that drones are cheap, and the prices of the necessary drones show a decreasing tendency, the investment into drone powered solutions is solid and advisable.

The use of IoT device networks has not yet become a standard solution in agricultural practice, as it was expected initially. At the beginning of their advent, specific solutions were only used experimentally on small plots of land and hothouses. The most often cited reasons for their limited implementation include the fixed location of sensors, their high price and the large number of required devices leading to large investments, especially for larger areas.

The purchase of drones must be preceded by careful needs analysis. Fixed wing drones can best be used for long plots of land without significant, quickly and suddenly appearing obstacles over the plots. Fixed wing drones are fast, but therefore also difficult to steer, and more importantly, they require considerable space for their flying maneuvers. Copters, on the other hand, are much lighter and agile, they can float in one spot over the plot, which is impossible for fixed wing drones. For this reason, copters are more suitable for smaller plots of land and/or plots in hard to reach areas (not 
accessible for fixed wing drones), as well as for longer continual monitoring of a certain process on a given part of the land area. Some farmers and almost all operators/providers of crop monitoring services using drones purchase and both of these two types of drones: for large plots of land with long linear aerial passages and no obstacle in the flight path, they use fixed wing drones, while for spot-checking or small, problematic parts of plots and/or monitoring of surface that are inaccessible for fixed wing drones, they use copters.

In general terms, implementation of IoT systems can be relevant for those who:

- own multiannual plantations (forests, vineyard, orchards),

- own smaller plots of land with highly profitable yield (legal plantations of marijuana in the USA),

- own large properties where the following characteristics are identical or very similar across the entire area: altitude, surface flatness, soil composition, wind rose, hydro-meteorological conditions, environmental impact (requiring a smaller number of IoT devices), and finally,

- are ready to make larger investments.

Drones are likely to be implemented by organizations and farmers who:

- own large properties with crop rotation and dynamic change of crop plot sizes for individual crops,

- own large properties where the values of soil composition and surface flatness, altitude and environmental impacts, as well as the hydro-meteorological conditions are different across the entire property,

- are not ready to, or could not afford to make larger investments, and finally,

- provide $S^{2}$ aaS services; as these services have greater possibilities for implementation if they are realized with drones, as opposed to IoT

systems/devices, because their field of monitoring can be dynamically changed.

In the authors' opinion, however, the $\mathrm{S}^{2}$ aaS services via IoT systems are not

likely to be significant in agriculture.

The spectrum of drone implementation is wide and is likely to expand so as to include even more fields of application. As a short indication of this trend, three interesting areas of using drones are given below.

The startup 'BioCarbon Engineering' has a prototype of a transformed combat UAV that is used in the service of tree planting with a $75 \%$ uptake rate, as opposed to the uptake rate of $8 \%$ for dispersing dry seeds by air - this technique could reduce costs of the traditional methods by up to $85 \%$ ! The field of implementation includes "planting in remote or hazardous areas, such as those affected by fires or chemical spills, ...there are types of trees that absorb contaminating elements, thus clearing the soil and making it suitable for further seeding." (Mikhailova, 2016).

Researchers in Japan have created small insect-sized drones which can pollinate plants. This will assist with indoor pollination and assist the real honeybee population (Chechetka et al., 2017; Evans, 2017).

And as a final example, wild monkeys in Oita Prefecture in Japan are dispersed and kept away from agricultural properties with the help of drones disguised as hawks. $(* * *, 2017 b)$. 
Petkovics, I.; Petkovic, D. \& Petkovics, A.: IoT Devices vs. Drones for Data Collec...

\section{Conclusion}

All state-of-the-art agricultural subjects are faced with the problem of data collection and processing. These activities need to be efficient, sometimes real-time, while also cheap. This chapter offers two alternatives for solving this problem: the IoT system and the drone-powered solution. The main conclusion is that IoT systems and drone-powered solutions are capable, suitable and successful in data collection and data processing in agriculture. Their indispensable services, defined on IoT platforms, realized via the cloud, Edge computing or Fog computing are ensured by the end product, which is the main focus of the end users: vital and useful information for running farms and organizations in the agricultural world.

Aspects to be considered whether to opt for IoT systems or unmanned aerial vehicles for data collection and data processing in agriculture are elaborated in the Discussion section and are intended as guidelines for making such a decision. As a third alternative for these tasks, there is the option of drone-powered $\mathrm{S}^{2} \mathrm{aaS}$. This field is likely to be an area of intensive research in the future.

\section{References}

Chechetka, S. A., Yu, Y., Tange, M. \& Miyako, E. (2017). Materially Engineered Artificial Pollinators, Chem, Vol. 2, No 2, (February 2017) 224-239, ISSN

Corrigan, F. (2017). Multispectral Imaging Camera Drones In Farming Yield Big Benefits, May 29 2017, Available from: https://www.dronezon.com/learn-aboutdrones-quadcopters/multispectral-sensor-drones-in-farming-yield-big-benefits/,

Accessed on: 2017-07-13

Davies, R. (2015). The Internet of Things - Opportunities and challenges, European ParlimentaryResearch Service, May 2015, Available from: http://www.europarl.europa.eu/RegData/etudes/BRIE/2015/557012/EPRS_BRI(2015 )557012_EN.pdf, Accessed on: 2015-09-24

Delaporte, E. (2015). An introduction to agricultural UAV imaging systems, October 7 2015, Available from: https://www.linkedin.com/pulse/introduction-agriculturaluav-imaging-systems-ed-delaporte? articleId $=8180971362733914695$, Accessed on: 2016-03-17

Evans, K. (2017). Tiny Robot Drones Developed To Help Declining Bees Pollinate Plants, Available from: http://www.iflscience.com/technology/tiny-robot-dronesdeveloped-to-help-declining-bees-pollinate-plants/, Accessed on: 2017-08-07

Grgurevic, I.; Perakovic, D.; Forenbacher, I. \& Milinovic, T. (2015). Application of the Internet of Things Concept in Carsharing System, Chapter 34 in DAAAM International Scientific Book 2015, pp.401-414, B. Katalinic (Ed.), Published by DAAAM International, ISBN 978-3-902734-05-1, ISSN 1726-9687, Vienna, Austria Konsek, H. (2015). The Architecture of IoT Gateways, August 18 2015, Available from: https://dzone.com/articles/iot-gateways-and-architecture, Accessed on: 201707-17 
Libelium, (2017a). Over the Air Programming (OTAP), Available from: http://www.libelium.com/products/waspmote/ota/, Accessed on: 2017-07-08

Libelium. (2017b). 50 Real IoT success stories after ten years of experience in the market, Available from: http://www.urenio.org/2017/07/20/libelium-white-paper-50real-iot-success-stories-ten-years-experience-market/, Accessed on: 2017-08-08

Linthicum, D. (2017). Three Types of IoT Data Sources, Available from: https://www.rtinsights.com/three-types-of-iot-data-sources/, Accessed on: 2017-08-10 Mikhailova, S. (2016). Using Ex-military Drones to Plant Trees. Available from: http://www.huffingtonpost.com/pro-journo-using-ex-militatydrones_b_8414532.html, Accessed on: 2017-08-13

Nebiker, S., Lack, N., Albacherli \& Laderach S. (2016). Light-weight multispectral uav sensors and their capabilities for predicting grain yield and detecting plant diseases, July 2016, Available from: https://www.int-arch-photogramm-remote-sens-spatial-infsci.net/XLI-B1/963/2016/isprs-archives-XLI-B1-963-2016.pdf, Accessed on: 201707-15

Nixon A. (2016). How To Select an Agriculture Drone: An In-Depth Buyer's Guide, Available from: http://bestdroneforthejob.com/drones-for-work/agriculture-dronebuyers-guide/ , Accessed on: 2016-03-24

Nixon, A. (2017). Best Drones For Agriculture 2017: The Ultimate Buyer's Guide, Available from: http://bestdroneforthejob.com/drones-for-work/agriculture-dronebuyers-guide/, Accessed on: 2017-08-15

Noyen, K.,Volland, D., Wörner, D \& Fleisch, E. (2014). When Money Learns to Fly: Towards Sensing as a Service Applications Using Bitcoin, September 2014, Available from:

https://www.researchgate.net/publication/265966871_When_Money_Learns_to_Fly_ Towards_Sensing_as_a_Service_Applications_Using_Bitcoin, Accessed on: 2017-0717

Pardey P., Beddow J., Hurley T., Beatty T. \& Eidman V. (2014). A Bounds Analysis of World Food Futures: Global Agriculture Through to 2050. Australian Journal of Agricultural and Resource Economics, Vol. 58, No. 4, (October 2014) pp (571-589) Perera, C. (2017). Sensing as a Service for Internet of Things: A Roadmap. Leanpub Publishers

Perera, C., Qin, Y., Estrella, J.C., Reiff-Marganies, S. \& Vasilakos, A.V. (2017). Fog Computing for Sustainable Smart Cities: A Survey. ACM Computing Surveys, Volume 50., Issue 3., (August 2017), Article No. 32

Petkovics, I. \& Petkovics, A. (2014). ICT Ecosystem for advanced Higher Education, Proceedings of 2014 IEEE 12th International Symposium on Intelligent Systems and Informatics (SISY 2014), Szakál, A. (Ed.), pp. 181-185, ISBN 978-1-4799- 5997-6, Subotica, Serbia, September 2014, IEEE

Petkovics, I. \& Tumbas, P. (2014). A felhőinformatika szerepe a XXI. Századi felsőoktatásban, In: A felsőoktatás tudományos, módszertani és munkaerőpiaci kihívásai a XXI. században, Mészáros, A., (Ed.), 187-196, Széchenyi István Egyetem, ISBN 978-615-5391-32-3, Györ, Hungary (in Hungarian) 
Petkovics, I., Simon, J., Petkovics, A., \& Covic Z. (2017). Selection of Unmanned Aerial Vehicle for Precision Agriculture with Multi-criteria Decision Making Algorithm, Proceedings of 2017 IEEE 15th International Symposium on Intelligent Systems and Informatics (SISY 2017), (Ed.), pp. , ISBN, Subotica, Serbia, September 2017, IEEE (accepted paper)

Petkovics, I., Trninić, J. \& Đurković, J. (2013). The Role of Information Technology Support in Sustainable Development, Strategic Management, Vol. 18, No 4, December 2013., pp. 3-13, ISSN: 1821-3448, ISSN 2334-6191 (Online)

Zohu H. (2012). The Internet of Things in the Cloud - A Middleware Perspective, Taylor \& Francis Group, ISBN 978-1-4398-9302-9, Boca Raton Florida

*** (2009) Internet of Things: An Action Plan for Europe, European Economic and Social Committee Commission of the European Communities, Available from: http://www.eesc.europa.eu/our-work/opinions-information-reports/opinions/internetthings-action-plan-europe, Accessed on: 2017-06-24

$* * *$ (2016a). Clarity from above - PwC global report on the commercial application of drone technology, Available from: https://www.pwc.pl/pl/pdf/clarity-from-abovepwc.pdf, Accessed on: 2017-08-12

*** (2016b). OpenFog Architecture Overview, White Paper, OpenFog Consortium Architecture Working Group, Available from: https://www.openfogconsortium.org/wp-content/uploads/OpenFog-ArchitectureOverview-WP-2-2016.pdf, Accessed on: 2016-06-21

*** (2016c). Fog vs Edge Computing, Nebbiolo Technologies, Available from: https://www.nebbiolo.tech/wp-content/uploads/whitepaper-fog-vs-edge.pdf, Accessed on: $2017-08-12$

*** (2016d). The Drone Market Environment Logo List 2016, Drone Industry Insights, Available from: https://www.droneii.com/project/drone-market-environment-logolist-2016, Accessed on: 2017-07-19

*** (2017a) Xcraft X Plusone, Available from: http://xcraft.io/x-plusone-drone/, Accessed on: 2017-03-24

*** (2017b). Oita deploys drone disguised as hawk to scare off farm-raiding monkeys, Available from: https://www.japantimes.co.jp/news/2017/08/19/national/oitadeploys-drone-disguised-hawk-scare-off-farm-raiding-monkeys/\#.WZ7JIvgjHIU, Accessed on: 2017-08-22 